\title{
User Experience Design in Professional Map-Based Geo-Portals
}

\section{Bernd Resch $^{1,2,3, *}$ and Bastian Zimmer ${ }^{4}$}

1 Department of GIScience, University of Heidelberg, Berliner Strasse 48, Heidelberg 69120, Germany

2 Center for Geographic Analysis, Harvard University, 1737 Cambridge Street, Cambridge, MA 02138, USA

3 SENSEable City Lab, MIT, 77 Massachusetts Avenue, Cambridge, MA 02139, USA

4 Institute for Geoinformatics and Remote Sensing, University of Osnabrueck, Barbarastrasse 22b, Osnabrueck 49076, Germany; E-Mail: bzimmer@uni-osnabrueck.de

* Author to whom correspondence should be addressed; E-Mail: bernd.resch@ geog.uni-heidleberg.de; Tel.: +49-6227-54-5573; Fax: +49-6221-54-4529.

Received: 30 August 2013; in revised form: 25 September 2013 / Accepted: 8 October 2013 / Published: 24 October 2013

\begin{abstract}
We have recently been witnessing the growing establishment of map-centered web-based geo-portals on national, regional and local levels. However, a particular issue with these geo-portals is that each instance has been implemented in different ways in terms of design, usability, functionality, interaction possibilities, map size and symbologies. In this paper, we try to tackle these shortcomings by analyzing and formalizing the requirements for map-based geo-portals in a user experience based approach. First, we propose a holistic definition the term of a "geo-portal". Then, we present our approach to user experience design for map-based geo-portals by defining the functional requirements of a geo-portal, by analyzing previous geo-portal developments, by distilling the results of our empirical user study to perform practically-oriented user requirements, and finally by establishing a set of user experience design guidelines for the creation of map-based geo-portals. These design guidelines have been extracted for each of the main components of a geo-portal, i.e., the map, the search dialogue, the presentation of the search results, symbologies, and other aspects. These guidelines shall constitute the basis for future geo-portal developments to achieve standardization in the user-experience design of map-based geo-portals.
\end{abstract}


Keywords: map-based geo-portals; usability; user experience; empirical survey; design guidelines

\section{Introduction}

As per the INSPIRE directive [1], which entered into force in 2007, the member states of the European Union (EU) have to provide their environmental geo-data in a defined standardized format. Additionally, many countries have realized the value of a harmonized spatial data infrastructure (SDI) and the value of spatial data for economic benefits [2]. Consequently, we have recently been witnessing the growing establishment of map-centered web-based geo-portals on national, regional and local levels for professional use of geo-data.

A particular issue with these geo-portals is that each instance has been implemented in different ways in terms of design, usability, functionality, interaction possibilities, map size and symbologies. On the one hand, this heterogeneity in geo-portal developments is rooted in geospatial companies' unique designs; on the other hand, and more importantly, it is due to lacking consistent guidelines for implementing map-based geo-portals. This also results from the fact that the term "geo-portal" has not been sufficiently defined so far.

Currently, most developers of geo-portals focus on functionality and technology rather than on the user and their needs. This technology-centered approach often assumes that mature and progressive technology can compensate for deficient usability [3]. In most cases, the future users and the anticipated use of the developed application are not included in the design and development process. This is mainly due to the large endeavors in terms of time and financial means, which this method would induce [4].

As a result of these uncoordinated development efforts, only few surveys have been carried out that focus on map-based web pages and their requirements [5] and this is particularly true for web-based geo-portals. Consequently, experiences in assessing the specific requirements of map-based geo-portals are rare. Particularly, no overall study has been carried out so far that considers all key aspects of map-based geo-portals (map, search, presentation of search results, symbologies, and other aspects). In fact, previous approaches lack the investigation of the whole breadth of the topic of map-based geo-portals. That is why no consistent and uniform guidelines have been developed so far.

This paper aims to tackle these research shortcomings by analyzing and formalizing the requirements for professional map-based geo-portals. This is done by defining the functional requirements of a geo-portal, by analyzing previous geo-portal developments, by conducting an empirical user study to perform practically-oriented user requirements, and finally by establishing a set of user experience design guidelines for the creation of map-based geo-portals. Thus, in contrast to previous developments, this paper presents a user-centered approach instead of a technical development procedure driven by functional capabilities.

The research questions, which are addressed within our research, are defined as follows.

- How do previous geo-portal definitions differ, and which aspects does a holistic definition have to comprise? 
- What are essential user requirements for professional map-based geo-portals?

- Which user experience design guidelines need to be followed to comply with these requirements?

The structure of this paper is as follows: This introduction is followed by a section on related work in the area of map-based geo-portals. Section 3 presents the methodological background including a definition of the term "geo-portal" and a discussion of usability vs. user experience. Thereafter, Section 4 discusses the performed user study, which addressed reasonably experienced professional geo-portal users. In Section 5, we present the design guidelines and user experience criteria for map-based geo-portals that have been extracted as a result from the survey. Finally, Section 6 wraps up the paper with a conclusion.

\section{Related Work}

Despite the widespread use of map-based web pages, the number of studies that address the usability of such sites is relatively low. Some of the conducted studies are mainly concerned with commercial mapping sites such as Yahoo Maps or Google Maps, as elaborated in the following paragraphs. However, the geo-portal as a map-based web platform is not explicitly considered in most studies.

One of these studies has been done by [6] who examine the needs and expectations of users in map-based web pages. The study focuses on users without any Geographical Information Systems (GIS) experience, but ignores the inclusion of GIS experts. The choice of participants for the study seems dedicated to the particular study contents, as the individuals have often used commercially operated map-based web pages before. In contrast, our study also considers somewhat experienced users of map-based applications because the primary usage context of geo-portals is professional work (see Sub-Section 4.2).

The work of Hornbaek et al. [7] primarily focuses on a particular component of map-based web sites, namely the overview map. The authors analyze the impact of an overview map on user interaction. Similarly, the studies by Harrower and Sheesley [8] and You et al. [9] focus on selected tools for navigation including various ways of navigating in the map and zooming. As shown in these studies, the design of the user interface has a significant effect on the usability of the platform. One essential shortcoming of these approaches is that no overall analysis is carried out taking into account all key aspects, i.e., the described approaches lack the investigation of the whole breadth of the topic of map-based geo-portals, which our study aims to do (see Sub-Section 3.3).

The study of Nivala et al. [5] goes one step further in investigating various commercial map-based services. The probands had to work on different tasks using various portals. At the end, 403 problems have been identified, which arose throughout the work with the different platforms. In addition to the identified deficiencies, a few proposals for solving such problems and to improve the usability were presented. The number of detected errors emphasizes how important the analysis of map-based web sites is. Our approach targets this necessity by the integration of user experience concepts additionally to well-known usability criteria.

The study by Kramers [10] shows that many users are unable to handle the mass of functionalities provided by map-based applications. The reason for this is the current focus on technology-oriented approaches, which do mostly not include the profiles of future users into the design and development 
processes. Consequently, many functions are developed from the point of view of GIS experts and are not suitable for the majority of the users. In contrast, the user-centered approach presented in this paper represents a useful way to avoid the most common mistakes made by users, and at the same time increases efficiency, effectiveness and satisfaction by taking into account clearly qualified user requirements.

A recent study that explicitly addresses geo-portal usability was performed by He et al. [11]. The national geo-portal of Sweden was investigated by a survey including a total of 14 test persons. Eleven tasks had to be processed with the help of the geo-portal. In addition, a user study to determine the probands' satisfaction was conducted. The two sub-components— "map" and "search"-were considered in more detail in this study. Only two of the eleven tasks were successfully completed during the investigation, although the Swedish geo-portal has been developed focusing on supporting these tasks. Furthermore, it is noted that many of the identified problems and errors could have been avoided in advance. Accordingly, the study proposes in the future work section the development of a guide with which the most common and best-known error can be avoided. Our work extends this approach by integrating the concept of user experience and by performing a study involving qualitative interviews with persons who use geo-portals in their professional life. Furthermore, we aim to establish a set of user experience design guidelines for map-based geo-portal development.

\section{Methodological Background}

This section lays out the methodological background for our work in terms of a definition of the term "geo-portal" (Sub-Section 3.1), a clear disambiguation of usability and user experience in the context of map-based geo-portals (Sub-Section 3.2), and finally the explanation of design dimensions for map-based geo-portals (Sub-Section 3.3).

\subsection{Geo-Portal Definition}

Spatial data have recently gained importance in a variety of business, public administration and research fields as it provides significant added value for various geographically-related operations. Many of these spatial data sets are owned by public authorities and institutions. With the INSPIRE directive [1] coming into force in March 2007, environment-related spatial data that are distributed over EU countries can be used across national and international boundaries as the EU member states are compelled to make their spatial data available in an INSPIRE-conformant way. As a result, several national geo-portals have emerged that provide spatial base data and information. Within these geo-portals, users are able to search for particular geo-datasets by looking into the associated metadata [12]. The application areas for such geo-portals are versatile including the identification of conservation areas, urban planning, traffic management, or planning environmental projects.

Generally speaking, a geo-portal needs to be distinguished from other spatial information retrieval mechanisms on the web. For instance, geographic information is also a central enabler of Internet-based mapping applications like Google Maps or Yahoo Maps. However, while these applications only serve for data portrayal, geo-portals offer the possibility to retrieve spatial data for further analysis. A further difference is that these specialized mapping services do not focus on integrating official geo-data such as, e.g., land use classifications or designated flood areas. Additionally, they do not provide access to spatial data via standardized service interfaces such as the Web Map Service (WMS) or the Web 
Feature Service (WFS) by the Open Geospatial Consortium (OGC). Consequently, these map-based web sites are not to be regarded as "geo-portals".

From a data retrieval viewpoint, it can be stated that pure search engines cannot be considered geo-portals as data queries are only one essential part, but do not make up a geo-portal as a whole. For instance, a geo-data search application, which allows users to discover spatial datasets, retrieve their metadata and finally access the data, is technically not a geo-portal as the map interface for user interaction and data portrayal is lacking.

Summarizing, it can be concluded that a geo-portal is a web-based system that allows users to discover particular geo-datasets by looking into the associated metadata, to portray the data on a map, and to retrieve the data in adequate formats to further process them in a professional workflow. The map itself shall constitute the central component of a geo-portal user interface in that is serves for geographic data discovery, for data display, and potentially for data retrieval using service-based data access approaches.

\subsection{User Experience vs. Usability}

This sub-section elaborates on the concepts of usability (Sub-Section 3.2.1) and user experience (Sub-Section 3.2.2), and shows how they differ (Sub-Section 3.2.3). This well-defined distinction between the two concepts also clearly shows how our approach differs from previous work and how it develops the current state of the art further.

\subsubsection{Usability}

Even more than for other geo-applications, usability is an essentially important aspect for map-based geo-portal applications. This is due to the fact that user interaction with a geo-portal involves a rather complex workflow including a variety of tasks including textual search, geo-search, result interpretation, data retrieval or map interaction, and that geo-portal users are potentially only conditionally experienced in using digital maps.

Usability generally constitutes a non-atomic quality criterion that describes how simply and intuitively user interfaces can be interacted with [13]. Usability is defined in the ISO 9241-11 standard as the "extent to which a product can be used by specified users to achieve specified goals with effectiveness, efficiency and satisfaction in a specified context of use" [14]. Nielsen's [13] original definition contained three more aspects as a part of system acceptability: learnability, memorability, and errors.

\subsubsection{User Experience}

In contrast to usability, which pursues a product-oriented approach that aims for technological optimization of user interfaces, user experience considers all aspects of user interaction. This means that while usability only tackles interaction issues during use of a product, user experience takes all aspects of before (anticipated use), during (actual use) and after (post-analysis of use) a product's use into account. In fact, usability is a part of user experience, which in turn means that good user experience cannot be achieved solely by good usability [15]. 
In the ISO standard 9241, part 210 on "Human-centered Design for Interactive Systems" [16], user experience is defined as "a person's perceptions and responses that result from the use and/or anticipated use of a product, system or service“. This definition includes all emotions, perceptions, preferences, as well as physiological and psychological reactions before, during and after use.

In other words, the concept of user experience involves a system's functionality, information portrayal, performance, interactive behavior, and the support of a user's physical and psychological state related to their experiences, attitudes, skills, personality and usage context $[17,18]$.

\subsubsection{Conclusion User Experience vs. Usability in Map-Based Geo-Portals}

From the differences in the definition of the terms "usability" and "user experience" it becomes evident that solely considering usability criteria in the technical design of map-based geo-portals is not enough to fulfill the user's needs. User experience comprises all the experiences, desires, expectations and opinions of users in dealing with a geo-portal whereas usability is just a set of technical guidelines for implementing such a portal.

Thus, we performed a user study to identify concrete user requirements including the usage context, tasks to be performed, reasons for using geo-portals, map requirements, necessary search possibilities, and potential improvements of existing geo-portals (see Sub-Section 4.2). This survey covers all three areas as defined in user experience, i.e., before use, during use and after use. From the results of the requirements analysis, we generated a set of design guidelines for the integration of user experience techniques into geo-portal design and evaluation processes (see Section 5).

\subsection{Design Dimensions for Map-Based Geo-Portals}

According to previous research efforts, we extracted five essential components of map-based geo-portals, which are described in the following paragraphs. These descriptions are intended to give a brief overview about the single aspects and are thus kept as short as possible. Sub-Section 5.2 contains a more detailed description that dedicatedly focuses on the actual definition of concrete user experience design criteria for map-based geo-portals.

The map is an essential part in a geo-portal as it is used for navigation and for the display of spatial data. Important aspects of the map are its size, resolution, information density, interaction possibilities, functionality, customizability to the users' particular needs, and performance in loading spatial datasets [19].

Apart from the map, search dialogues are another essential component for the effective use of the data in a geo-portal. In general, users should be able to choose between different search possibilities to find the matching spatial data or data services for their respective tasks. The most widely implemented search dialogues are:

- Free-text search using a text box.

- Spatial search over a geographical area.

- Category-based search.

The next essential functionality of a geo-portal is the presentation of the results of a user's search. Here, an adequate representation is crucial as a misleading presentation of the results can potentially 
cause misunderstandings in interpreting the given information [11]. This is particularly important as misinterpretations compromise the users' efficiency and effectiveness in performing their tasks.

Crucial aspects to consider in terms of the presentation of search results are the form of presentation, the display of metadata, the order of datasets, the use of multiple pages, font sizes, and further usage of the discovered datasets.

Symbols, i.e., icons for user interaction, are a central part of all web-based applications, and even more for map-based geo-portals. Symbols serve for accurately representing their hidden functionality in a graphical icon. This also facilitates the operation of the whole portal. Symbols can be optimised by complying with the following criteria: clarity, accuracy, simple interpretability, and visual feedback for the symbol that is currently in use.

Other aspects comprise long loading times, self-descriptiveness, communication between the user and the system, legends, pop-up windows, the use of advertising elements, "unexpected" effects, poor linking of the individual pages, the use of frames and scrollbars, animated graphics, inactive links, and, flashing and moving text.

\section{Empirical User Study}

To assess the requirements of map-based geo-portals and to fuse them with criteria defined in previous research, we carried out an empirical user study. This section presents the methodology and the results of the study. First, a short description of how the test persons have been selected is given in Sub-Section 4.1. Then, the results for each question are presented in Sub-Section 4.2 separately for each question, whereas the concept that has been developed based on the survey results is laid out in Section 5.

\subsection{Participants}

For the user study presented in this paper, we identified 164 people through an Internet-based search on web sites of geo-portal users including public bodies, local and regional governments, planning agencies, and companies, and contacted them by email and by telephone. We selected reasonably experienced users, i.e., persons who professionally and regularly use geo-portals. People who use geo-portals privately have not been included in the test group. This method has been chosen (1) because professionals have more experience in using geo-portals through their daily work; and (2) because geo-portals are mostly developed for professional users rather than for private users. Apart from geo-portal users, we also included geo-data and geo-service providers into the survey, as well from governmental institutions (federal states and communes) as from economy. Due to the country-wide spatial distribution of the test persons, they were contacted by email and asked to fill out an anonymous online survey using the survey platform SoSciSurvey (https://www.soscisurvey.de). The questionnaire was answered by 105 persons, where most of the people were older than 30 years.

The questionnaire comprised 20 questions in total: six closed, one hybrid and thirteen open questions. The large number of open questions has been chosen to optimize the trade-off between the large effort to conduct personal interviews, the value of a high number of participants, and the significant value of qualitative feedback. 


\subsection{User Study}

The questionnaire was divided into six categories that represent the five design dimensions defined in Sub-Section 3.3: general (Questions 1-6), map (Question 7), search (Questions 8-15), symbologies and tools (Questions 16-18), and personal (Questions 19,20).

\subsubsection{General}

Question 1 (open question) asked which geo-portals the participants are currently using. The question aimed to establish a list of currently used geo-portals, thus guaranteeing comparability of the results. The participants named 49 different geo-portal applications, where some of the probands named more than one portal (105 participants gave 177 answers). 89.8\% of all answers indicate the use of portals that are operated by public bodies (German geo-portal, Geoproxy Thuringia, Brandenburg Viewer, geo-portal Saxony, Bavaria Viewer, etc.), while 10.2\% of the answers contain other applications (Google Earth, Google Maps, OpenStreetMap, ArcGIS Online Explorer, etc.), which can partly not be considered a geo-portal as defined in Sub-Section 3.1.

Question 2 (open question) asked how frequently the test persons are using a geo-portal. This question's objective was to assess the frequency of use and hence to create an indicator for the importance of the geo-portal for the user's professional tasks. Approximately three quarters of the test persons (76.9\%) use geo-portals several times per week, $23.1 \%$ use it weekly. These figures show that the selected probands are mostly experienced geo-portal users in whose daily routines geo-portals play a central role.

Question 3 (open question) asked what the reasons are for using a geo-portal. The question aimed at getting information about the reasons for using a geo-portal, e.g., job-related, private or other reasons. However, most probands' answers referred to the purpose of using geo-portals with a strong focus on performed tasks and used functionalities. Thus, the answers to Question 3 have been combined with those to Question 4.

Question 4 (open question) asked for what purpose the participants are using a geo-portal. In combination with Question 3, this question aimed to draw conclusions about the motivation to use a geo-portal, which is a crucial aspect for user experience design. Figure 1 shows the answers in a pie chart grouped according to similar categorizable answers. Figure 1 shows that most test persons use geo-portals for data retrieval and data access (50.4\%). Other purposes for using geo-portals comprise basic functionality (printing, measuring, routing, etc.), orientation (location, overviews), data analysis (comparison with other datasets, visual analysis), project reports, or "other" purposes (publication of geo-data, map view, map creation).

Question 5 (open question) asked which functions of a geo-portal the test persons are using. This question tried to find out which functions the participants use most frequently in their operational routines. This can possibly build the basis for establishing a core tool set for geo-portals. $30.9 \%$ of the test persons indicated that they mainly use the map interface in a geo-portal for displaying base maps (aerial images, satellite images, topographic maps, street maps) or thematic maps (public maps, land use, cadastral maps). Furthermore, 29.2\% use basic functionality (measuring, zooming, printing, object 
identification), $12.5 \%$ search for places and addresses, and 6.6\% use geo-data services. Other aspects include metadata retrieval, data provision and supporting the orders of planning-relevant documents.

Figure 1. Usage of a Geo-portal.

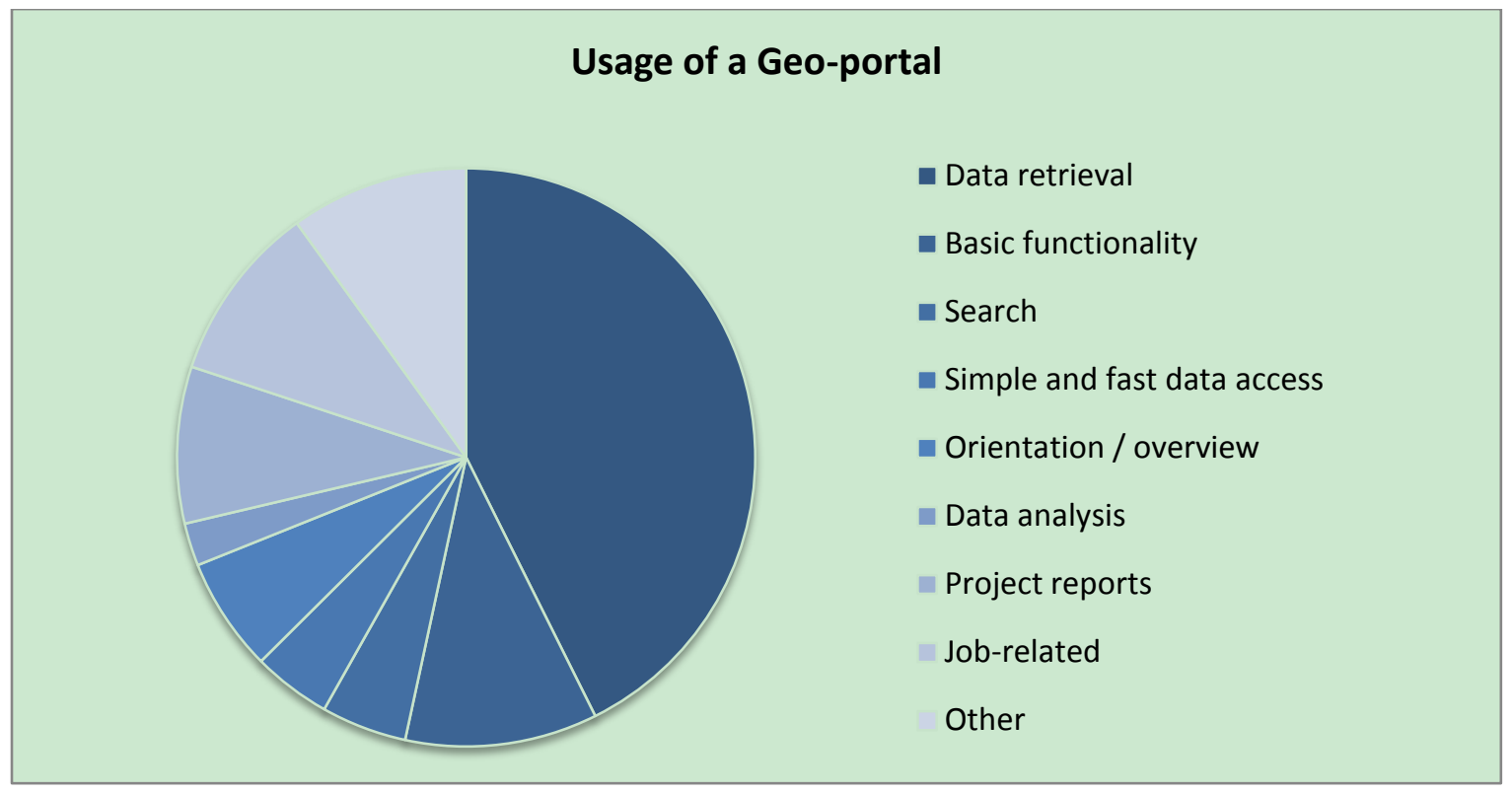

Question 6 (open question) asked what the first steps are after opening the geo-portal. As these steps are highly heterogeneous and dependent on the performed tasks, this question aimed to harmonize a certain operational procedure and thus, in turn, to optimize the design of the geo-portal and its user guidance. Figure 2 shows the answer distribution to Question 6. 51.3\% of the test persons use the search dialogue right after entering the geo-portal, while $20.9 \%$ use the map interface, $17.4 \%$ perform other steps (object identification, data retrieval, switch on the desired layer, etc.), $7.0 \%$ change their personal settings, and $3.5 \%$ try to get an overview about the portal and its functionality.

Figure 2. First Steps after Entering a Geo-portal.

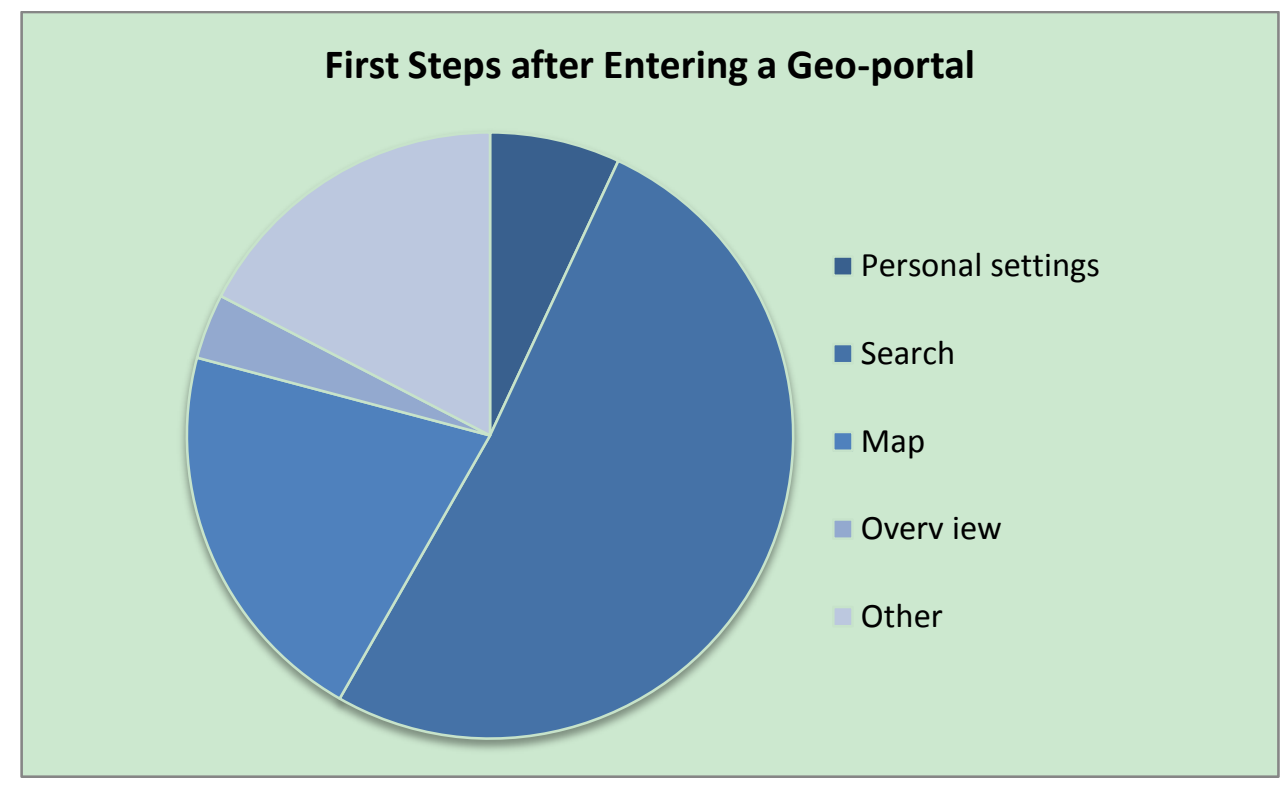




\subsubsection{Map}

Question 7 (yes-no question) asked whether the participants use the map of a geo-portal. This question was subdivided based on a test person's answer. In case the answer to the base question was "yes", three more questions (two open and one closed question) were asked about the purpose of using the map, about desirable improvements for the map presentation, and about the possibility of individual resizability of the map, according to the requirements, which we extracted from the literature review presented in Section 2. In case the answer to the base question was "no", two more open questions were asked on the reasons for not using a map and for needed improvements to increase the motivation for using a map. $87.1 \%$ of the participants answered the question with "yes", $12.9 \%$ answered with "no". The following paragraphs discuss the answers for each of the five sub-questions.

First, the three sub-questions for the case that the test persons answered the base question with "yes" are examined.

Purpose for using the map (open question): $23.3 \%$ of the test persons are using the map for visualizing thematic information (property borders, building blocks, general geo-information), 21.1\% for spatial orientation, $13.3 \%$ for creating project reports (building sites, effort estimation, land use, real estate benchmarks), $11.1 \%$ for basic functionality (measuring, geo-data overlays, digitization, printing), $8.9 \%$ for retrieving information, $7.8 \%$ for displaying a base map, and $14.4 \%$ for other purposes (data search, visual analysis, map creation, object search).

Desirable improvements of the map presentation (open question): $26.0 \%$ of the probands would like to see better performance of the map visualization, $20.0 \%$ more up-to-date and more accurate maps, $12.0 \%$ support for individual settings (customized legend, adaptability of the map's size, integration of external map layers), $8.0 \%$ printing functionality, 6.0\% additional map layers (basic maps, global map coverage, additional visualization possibilities), $4.0 \%$ advanced visualization of thematic geo-data, and $24.0 \%$ other aspects (larger map, better drawing functionality, attribute queries, information about data quality, support for all spatial reference systems).

Individual resizability of the map (closed question): This closed question asked whether geo-portal users should have the possibility of individually adapting the map's size. $76.5 \%$ of the study participants answered with "I fully agree", $17.7 \%$ with "I rather agree", $4.4 \%$ with "Undecided", $0.0 \%$ with "I rather disagree" and $1.5 \%$ with "I fully disagree".

Then, the two open sub-questions for the case that the test persons answered the base question (No. 7) with "no" are discussed.

Reasons for not using a map: $41.7 \%$ of the participants stated that the used map material is too inaccurate, $33.3 \%$ that they do not need a map for their work, $8.3 \%$ that the map data is too expensive, $8.3 \%$ that map data is stored locally on their computers, and $8.3 \%$ that maps are printed only in few cases.

Needed improvements: Desired improvements were only suggested by five participants. These improvements comprise easier ways to find the map in the geo-portal, cost-free provision of map data, harmonized geo-data, the possibility for search of districts, and the possibility for searching for area codes. 


\subsubsection{Search}

Question 8 (closed question) asked whether the test persons are satisfied are with the search dialogues in current geo-portals. In combination with Question 9, this question tried to assess the user's contentment with current implementations of search dialogues and whether they are easily usable. $16.0 \%$ of the test persons answered with "I fully agree", 52.0\% with "I rather agree", $25.3 \%$ with "Undecided", $6.7 \%$ with "I rather disagree", and $0.0 \%$ "I fully disagree".

Question 9 (closed question) asked whether the possibility of using several different search methods prevents the user from performing their actual working tasks. $1.5 \%$ of the participants answered with "I fully agree", $13.2 \%$ with "I rather agree", 14.7\% with "Undecided", $39.7 \%$ with "I rather disagree", and $30.9 \%$ "I fully disagree".

Question 10 (hybrid question) asked which search possibilities the test person prefers. The answers of this question can be used to optimize search possibilities. The three pre-defined answering possibilities to this hybrid question were "entry of keywords into a search field", "search via a geographic extent", and "search via categories". Additionally, the participants could enter a free-text comment into a text box. Figure 3 shows the answer distribution- $45.8 \%$ prefer the keyword search, $36.1 \%$ the geo-search, $15.3 \%$ the category search, and $2.8 \%$ other search possibilities (combination of keyword and geo-search, Google-like semantic search).

Figure 3. Preferred Way of Searching for Geo-data.

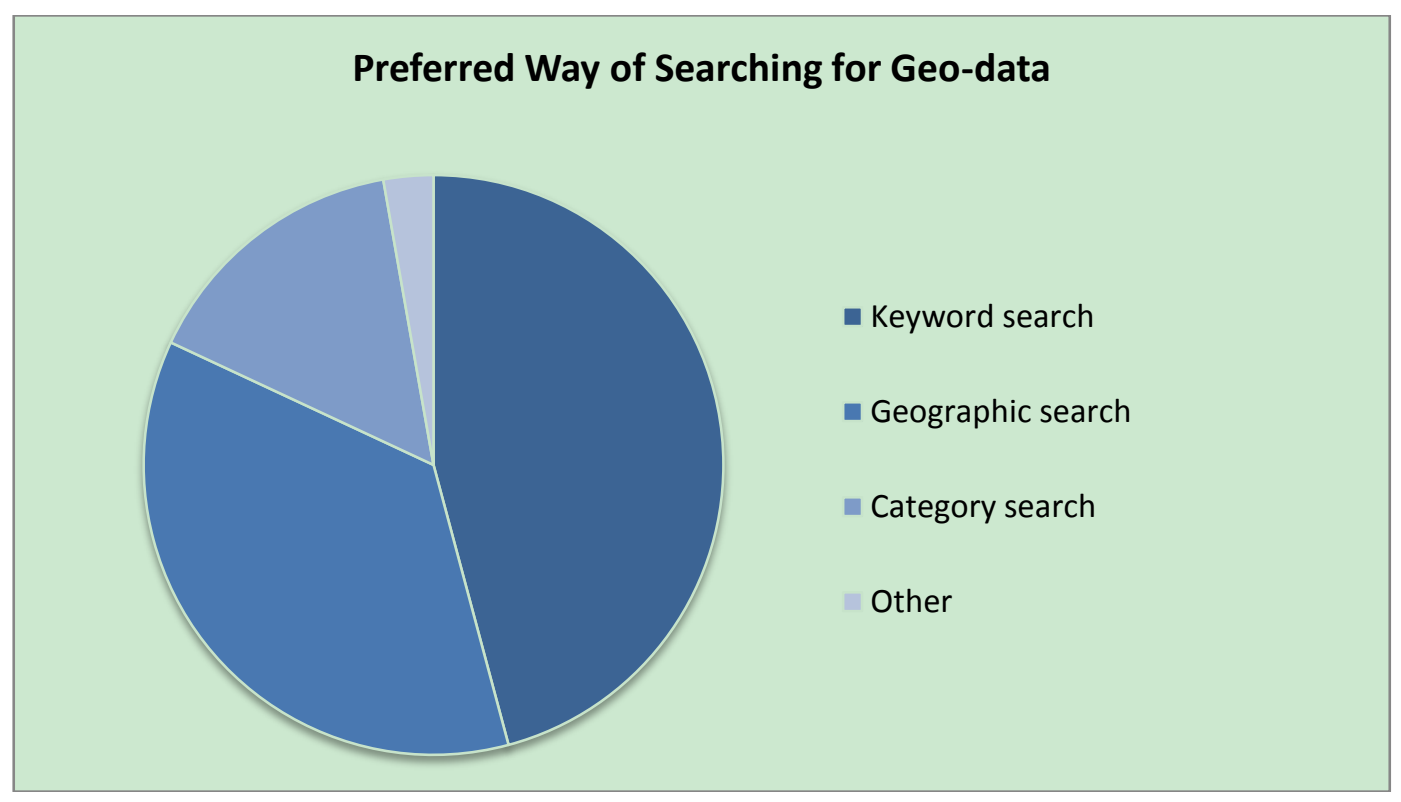

Question 11 (open question) asked what the users are usually looking for when performing a search in the geo-portal. In combination with Question 12, this question aimed to assess preferred search terms and potential improvements, which can then be used to enhance search algorithms and interfaces. 52.4\% of the test persons indicated that they search for thematic geo-data (land parcels, land use, property owners, zoning plans, cadastre), $23.8 \%$ for locations or addresses, $9.5 \%$ for real properties, $1.9 \%$ for geo-data services, and $12.4 \%$ for other objects (Points of Interest (POIs), persons, buildings, metadata, city maps). 
Question 12 (open question) asked what possible improvements they could imagine for the search dialogue in geo-portals. $39.4 \%$ of the probands stated that they would like to see improvements in the flexibility of the search (compass search, finding synonyms, flexible address search, ability to handle spelling mistakes, Google Maps-like search), 15.2\% in the availability of data sources (one input field that sends search request several data repositories, better connection between search results and base data, tree-like search), $9.1 \%$ in the performance (decrease of waiting times, faster data access), $9.1 \%$ in usability (user interface, more intuitive input possibilities, free-text search), and $27.3 \%$ in other aspects (active zooming, availability of geo-data services in the search, search history, phonetic search).

Question 13 (closed question) asked whether users think that the search results in current geo-portals are displayed in an easily-comprehensible manner. $25.0 \%$ of the participants answered with "I fully agree", $52.8 \%$ with "I rather agree", $20.8 \%$ with "Undecided", $1.4 \%$ with "I rather disagree", and $0.0 \%$ "I fully disagree". In combination with Question 15, this question tried to assess current shortcomings of the presentation of search results and subsequently optimize the illustration of these results to make the operational procedures more efficient.

Question 14 (open question) asked how the test persons would like the search results to be structured. $41.0 \%$ of the test persons would like to have the search results presented in tabular form (unambiguous, precise, structures, clear and categorized presentation), 28.2\% directly in the map (graphically as geographic map data with zoom to the result), $7.7 \%$ in individual visualization techniques (presentation according to screen size and the usage context), and $23.1 \%$ in other forms (uniform illustration, presentation in separate window, faster portrayal).

Question 15 (open question) asked which steps the participants perform after they have found a suitable result in the geo-portal. This question was dedicatedly asked because there is no standard workflow for using search results of geo-portals. The goal of the question is to find out about participants' operational procedures of using geo-data such as pure visualization, integration into a desktop GIS, provision over the Internet, export, or transformation into other data formats. $24.3 \%$ of the test persons answered that they display the results on a map, $23.0 \%$ print the results, $18.9 \%$ retrieve further information (metadata, object details, additional geo-data layers, retrieve related data), $13.5 \%$ integrate the results in their work procedures (measuring, save the data), 10.8\% analyze the results (geo-data evaluation, geo-analysis, selection of the most useful results), and $9.5 \%$ perform other tasks (call geo-data service, purchase the data, varying tasks depending on the current goal).

\subsubsection{Symbologies and Tools}

Question 16 (closed question) asked whether the current order and the placement of the symbols supports the test persons' tasks. $27.4 \%$ of the probands answered with "I fully agree", $48.6 \%$ with "I rather agree", $18.6 \%$ with "Undecided", $4.3 \%$ with "I rather disagree", and $1.3 \%$ "I fully disagree". In combination with Question 17, this question aimed to evaluate how the symbols and tools of current geo-portal implementations support users in performing their tasks.

Question 17 (closed question) asked whether it is clear to the participants, which functionality a symbol indicates. $11.9 \%$ of the test persons answered with "I fully agree", 32.8\% with "I rather agree", $37.1 \%$ with "Undecided", $13.4 \%$ with "I rather disagree", and $4.5 \%$ "I fully disagree". 
Question 18 (open question) asked how the use of symbols and tools could be improved. Following Questions 16 and 17, this question aimed to find out potential improvements for symbols and their order by collecting feedback as well from geo-portal users as from designers. $29.3 \%$ of the test persons stated that symbols should be more clear and self-explanatory (unambiguous, colored, large symbols using pictograms), $15.5 \%$ that symbols should be standardized, $15.5 \%$ that help should be offered in using the symbols (help button, detailed description in a separate help dialogue, tool-tips), 10.3\% that the number of tools and symbols should be reduced, $8.6 \%$ that the symbols should be individually customizable, $8.6 \%$ that the order should be improved, and $12.1 \%$ mentioned other aspects (Windows-like presentation, no nested functionality, adherence to common practice in current GIS software).

\subsubsection{Personal}

Question 19 (closed question) asked about the participant's age group. 9.6\% of the test persons answered with "below 30 years", 60.3\% with "30-50 years", and $30.1 \%$ with "over 50 years".

Question 20 (open question) finally asked, in which area the test person is working. 56.9\% work in public services and administration, $12.3 \%$ in planning offices, $10.8 \%$ in forestry and agriculture, $7.7 \%$ in public utilities (municipal utilities, waste management, energy supplier), and $12.3 \%$ in other areas (universities, self-employed, construction firms).

\section{Results: Design Guidelines and User Experience Criteria for Map-Based Geo-Portals}

This section condenses the results from the user study presented in Section 4 in the context of the user experience criteria described in Sub-Section 3.2, and suggests an extended core set of guidelines specific to the establishment of map-based geo-portals. These guidelines comprise two sub-parts: First, the definition of user experience parameters, and second the establishment of dedicated design considerations according to the five dimensions defined in Sub-Section 3.3.

\subsection{Result: User Experience for Map-Based Geo-Portals}

This sub-section proposes a definition of criteria that are relevant to user experience design for map-based geo-portals (see Sub-Section 3.2). To account for the three phases of user experience as described in Sub-Section 3.2 (before, during and after use), we performed the following tasks: (1) Before use: In our user study, we queried the particular requirements of potential users of professional map-based geo-portals; (2) During use: We established a number of usability-related criteria as described below; (3) After use: In the user study, we also asked geo-portal users about their experiences and potential improvements; this input serves for the integration of user experience techniques into geo-portal design and evaluation processes. The questions on before and after use have been asked in one combined questionnaire as the probands of the study all had reasonable experience in using geo-portals.

The following paragraphs lay out for each of the usability-related parameter (1) a general description of the criterion; and (2) how the parameter is essential to the concept presented in this paper. We mainly extracted the parameter descriptions from the survey presented in Section 4, but also accounted for outcomes of previous research efforts. 
Users: This parameter describes users or user groups in terms of their pre-knowledge, experience, usage context (physical, technical and social), age, etc. For map-based geo-portals, the typical user is 30-50 years old (independent of gender) and accesses the geo-portal in an office environment. The typical user has considerable experience in using maps and search functionality as the geo-portal is used several times a week. Furthermore, the user knows how to transfer information from one system to another in order to support their tasks.

Goals: This parameter defines which tasks a user performs with an application and which goals shall be achieved. For the presented geo-portal use case, goals and are manifold, but mostly involve geo-data. The performed tasks predominantly comprise data retrieval, data access and basic functionality like printing, measuring and routing. Other minor tasks comprise orientation, data analysis, publication of data, and the creation of project reports. In terms of functionality offered by a geo-portal, the typical user mostly displays the base maps (aerial images, satellite images, topographic maps, street maps) or thematic maps (public maps, land use, cadastral maps). Moreover, they use basic functionality (measuring, zooming, printing, object identification), the search for places and addresses, and geo-data services including metadata retrieval.

Context: This parameter defines the usage context composed of the physical context (physical environment, degree of mobility, etc.), the technical context (type of device, device capabilities, used software, etc.) and the social context (distractors, interaction with other persons, public or private environment, etc.). For the presented geo-portal use case, the user's context is typically an office environment that includes low background noise, little distraction and a well-equipped work space (a high performance work station, a large desktop screen, standard input devices like keyboard and mouse). Thus, users will usually be inside and not moving around, but sitting at their office desk. The user is typically without company, thus not implicitly sharing the interest of retrieving information and using the map-based geo-portal.

Learnability: Learnability indicates how easy it is for users to perform specific tasks the first time they use an application. For the concept presented in this paper this criterion is of minor importance as the typical user accesses the geo-portal several times per week, and has considerable experience in using the offered tools. However, many participants in our survey mentioned the need for clear and comprehensive help dialogues. Here, it is crucially important to find the optimal trade-off between a comprehensive textual introduction (too much textual information can potentially overburden the user and result in fading interest) and conveying as much information as possible. A solution to successfully reach this compromise is to use illustrations, which are enriched with short textual hints, to point the users to the essential core functions. Additionally, the design (including the presentation of the core functionality) of the geo-portal needs to be optimized. Aspects supporting this optimization are presented in Sub-Section 5.2.

Efficiency and Effectiveness: These parameters state how quickly (in terms of time and effort) and to what degree (how effectively) users can perform a certain task once they have established proficiency in using the application. As the map-based geo-portal use case gives the user enough time to comprehend the application and its functionality, and the geo-portal is used several times per week, the user interface's design can be more complex in comparison to other applications that are not as frequently used. Nevertheless, the portal in general and the map in particular have to adhere to common design guidelines with regard to optimized information density, consistent color schemes, 
minimal eye movement in performing standard tasks, and the minimization of the number of clicks to reach a certain goal [20]. Furthermore, a central feedback in the user study was the optimization of performance in the geo-portal. Thus, the presented concept suggests a caching mechanism in order to pre-load map data in the adjacent raster cells around the current position (in all directions). This results in a smooth experience in navigating through the map without the need to wait for the according location-dependent data. The same applies to high-performance search algorithms and the efficient presentation of the search results.

Memorability: Memorability defines how easy it is for users to re-establish their proficiency when they re-use an application after not having used it for a certain period. The criterion of memorability is of minor importance for map-based geo-portals as the survey results show that the typical user accesses the portal several times per week and has significant experience in using maps and geo-portals. Thus, the learning step will be minimal each time the user calls the portal application. In turn, this means that the parameters effectiveness, efficiency and satisfaction are of great importance for map-based geo-portals.

Errors: This parameter indicates how many errors users make and how these errors affect usage of an application, i.e., how users recover from these errors. In the context of map-based geo-portals, the typical user is considered experienced enough not to make trivial errors in using the map or the portal, and that they can cope with extraordinary situations (unusual reactions of the portal, inaccurate map data, unexpected results). One major concern in terms of errors is consistency in the search results and their presentation. As users increasingly expect intelligent ("Google-like") search, other forms of non free-text search and tabular presentation will be difficult to adapt to.

Satisfaction: Finally, satisfaction describes how subjectively pleasant it is for users to use the application. As the typical geo-portal user uses the application several times per week, satisfaction is of crucial importance to the presented concept. For the conception and implementation of a map-based geo-portal this means that geo-data need to be loaded into the map without delay, the interface has to be customizable to the user's individual needs, the map's size has to be adaptable, the geo-data need to be accurate, and users have to be able to find geo-data (including according meta-data) using the search dialogue. Furthermore, just like for the category of errors, one major concern in terms of satisfaction is consistency in search results and their presentation. This is due to the fact that users widely expect intelligent search, whereas other forms of non free-text search and tabular presentation are considered difficult to adapt to and thus decrease subjective satisfaction when using a geo-portal.

\subsection{Result: Design Considerations}

Apart from the user experience design criteria described in the previous sub-section, several additional design guidelines need to be considered when creating a map-based geo-portal. The following sub-sections contain a number of considerations for each of the five design dimensions as specified in Sub-Section 3.3 (map, search dialogue, presentation of search results, symbologies and other aspects). The design established set of considerations is based on the results of the user study presented in Section 4 and extends previous research, whose results are widely in line with ours, but only cover parts of our more broadly conceived study. 


\subsubsection{Map}

Our user study showed that most test persons use geo-portals for data retrieval and data access (50.4\%). To perform these tasks, the map is used for orientation purposes (location, overviews). Furthermore, nearly a third of the participating geo-professionals indicated that they mainly use the map interface in a geo-portal for displaying base maps (aerial images, satellite images, topographic maps, street maps) or thematic maps (public maps, land use, cadastral maps). These indications coupled with the fact that opening the map interface after entering the geo-portal is a standard procedure underpins the necessity of a thoroughly-designed and well-embedded map interface in a geo-portal.

A crucially important aspect is the interaction between the user and the map. In general, users perceive it more enjoyable to zoom by (double) clicking the map, instead of a selection of discrete zoom levels [21]. The same applies to navigation on the map: A drag-and-drop based approach for panning appears to be the more natural one in comparison to navigation buttons on the edge of the map. This is due to the facts that (1) the buttons have to be pressed more often to navigate over longer distances; and (2) clicking small buttons is difficult and makes navigation tedious. This suggests the use of the "contemporary" and modern drag-and-drop style of navigation, as this is offered by well-known mapping services such as Google Maps as today's geo-portal users are experienced in this kind of navigation. Furthermore, access time increases if users need to press different buttons for navigating in a certain direction, and navigation is restricted to predefined distances. This is of particular importance as the map's performance is a major user experience criterion.

Generally speaking, a larger map is advantageous to support users in their work [6,20]. Through a larger map, the user is less occupied with the interaction itself. Consequently, the user is more focused on the actual task and the workflow instead of cognitively demanding interaction. In order to make an approximate indication of the optimal size, most of the users of a desktop GIS use a map, which occupies about $70 \%$ of the screen [22]. Accordingly, approximately $70 \%$ of the available area should be covered by the map of a geo-portal.

In terms of resolution and dynamic information presentation, no digitized paper maps should be used as they are not suitable for the web due to lacking scaling of single elements. Another drawback of these maps is that the display likely clutters the user with too much information [5] as dynamic data sub-selection and scale-dependent clustering are not possible. This is particularly important because a central requirement for data portrayal on digital maps is zoom level dependent information density.

Another increasingly crucial aspect is the customizability of the map's size to the individual needs of users [23] $-94.2 \%$ of the study participants consider this an important feature. The situation of users' different screen sizes and resolutions leads to problems in that the operational flow is disturbed as the map size is oftentimes not adapted to these screen settings [11]. Thus, it would be beneficial to give the user the ability to customize the size of the map to their individual requirements.

Other important aspects regarding a geo-portal's map comprise the accuracy of the map's base data, simple ways to find the map in the geo-portal, harmonized geo-data, and the support for individual settings (customized legend or integration of external map layers). 


\subsubsection{Search}

Apart from the map, search dialogues are another essential component for the effective use of the data in a geo-portal. This is shown by the results of our user study, in which a majority of the participants indicated that they use geo-portals for data retrieval and data access. Furthermore, most participants stated that they use the search dialogue right after entering the geo-portal.

In general, users should be able to choose between different search possibilities and find the matching spatial data or spatial data services for their respective tasks. As mentioned above, the most widely implemented search dialogues are free-text search, spatial search, and category-based search.

The free-text search fields that are offered by most geo-portals spark certain expectations of the users as a search engine is a daily commodity for searching information on the Internet. Thus, users tend to use search dialogues in geo-portals in the same non-structured and "intuitive" way like web-search engines. Search requests have become increasingly longer in recent years [11], i.e., users are searching for long sentences rather than for short tags.

In this regard, the main problem is that most systems simply compare the entered search words with the stored metadata. Using this simple text comparison, an exemplary problematic search could be: "A nature reserve near Munich". In this case, the issue is that in most geo-portals, this request would not lead to a reasonable result because the "vague" nature of the search terms would not comply with the metadata associated to the particular datasets. In addition to fuzzy search terms, entering a long and complex query usually leads to errors. Yet, the search box should not be too small in order to facilitate the input of longer search queries. To define a concrete value for the size of the search field, the findings of Nielsen and Loranger [24] can be used. They recommend a text box length of at least 27 to 30 characters. In general, spelling errors can be minimized using a long search field as the query can be viewed as a whole.

Besides entering free text, data retrieval by specifying a geographic area is another search dialogue possibility for map-based geo-portals. In general, there are two methods for specifying a geographic area: (1) by manually entering coordinates; and (2) by specifying a spatial extent (e.g., a bounding box) using the mouse. A central issue with entering coordinates is that users often enter various place names instead of the required coordinates. Consequently, users need to be made aware of the correct use, for instance, by setting sample values in the search fields.

Despite the possibility of offering different possibilities for discovering geo-data, it needs to be considered that providing several different search methods potentially prevents the user from performing their actual working tasks $-70.6 \%$ of the study participants indicated this. Currently, text-based search is still the preferred way of data discovery $(45.8 \%$ of our test persons prefer the keyword search, $36.1 \%$ the geo-search, $15.3 \%$ the category search), but it needs to be thoroughly conceived and implemented considering the high expectations to free-text search engines that are common in web search engines. Yet, $52.4 \%$ of the test persons indicated that they search for thematic geo-data (land parcels, land use, property owner, zoning plans, cadastre) and $23.8 \%$ for locations or addresses. This suggests that the spatial search can be a valuable tool for map-based geo-portals as well. In addition, the provision of a search history to geo-portal users significantly enhances productivity.

Summarizing, it can be stated that flexibility of the search including compass search, finding synonyms, flexible address search, ability to handle spelling mistakes, Google Maps-like search, etc. is 
a central requirement $39.4 \%$ of the probands stated that they would like to see improvements in the flexibility), regardless of the search method. Other desired improvements comprise easier ways to find the map in the geo-portal, a search history, cost-free provision of map data, harmonized geo-data, the possibility for search of districts, and the possibility for searching for area codes.

\subsubsection{Search Results}

As stated in the previous sub-section, most of our study participants use geo-portals for data retrieval and data access including the analysis of metadata. After having found the appropriate data, users predominantly display the results on a map, print the results, retrieve further metadata, integrate the results in their work procedures, or analyze the results.

Thus, apart from adequate search capabilities, the presentation of the search results is a central aspect in designing map-based geo-portals. Most of the test persons of our user study would like the search results to be structured $-41.0 \%$ of the test persons would like to have the search results presented in tabular form (unambiguous, precise, structured, clear and categorized presentation) and $28.2 \%$ directly in the map (graphically as geographic map data with zoom to the result).

In general, an adequate representation is crucial as a misleading presentation of the results can cause misunderstandings in interpreting the given information [11]. A common agreement is the presentation of search results in a tabular form. However, the respective metadata should always be displayed in addition, and a reference to the map should be offered, e.g., in the form of a clickable link, through which the result can be displayed on the map. Furthermore, the possibility of sorting or comparing the results should be provided [3], where datasets can, for instance, be sorted by date or by relevance.

In terms of the visual presentation of the search results, displaying content on multiple pages should be avoided. Otherwise, geo-portal users will often not be able to identify search results on consecutive pages. In case of displaying results on several pages, the references to other pages have to be instantly recognizable and their purpose needs to be clear. In addition, the references should be positioned at the top of the site [4].

More, the descriptive text complementing the actual geo-data should be easy to understand and accurate, and complicated technical terms should be reduced. For better readability, the text should not be too stocky and have a minimum font size of ten points. The actual font size depends on the selected font [24]. A sans-serif font should be used preferentially for a better readability.

Another important aspect is the design of a user interaction dialogue if no matching result is found. In this case, constructive feedback from the geo-portal is needed. The message that "no results could be found" is not a productive type of feedback because it frustrates the user and leaves them back without other ideas for search terms. Thus, a list of possible alternatives would be a better way to deal with an empty result set. In addition, according to Nielsen and Loranger [24], the feedback in the form of a blank white page should be avoided.

\subsubsection{Symbols}

Symbols, i.e., icons for user interaction, are a central part of all web-based applications, and even more for map-based geo-portals. Symbols serve for accurately representing their incorporated functionality 
in a graphical icon. This also facilitates the operation of the whole portal. Symbols can be optimized in the following ways:

- The symbol should be clear and accurate.

- The symbol should be easily and correctly interpretable without additional assistance.

- The symbol should give visual feedback if it is currently in use.

Most of the current geo-portals do not fulfill these aspects, as icons are oftentimes not legible and lead to misinterpretations. Generally speaking, implementing unambiguously interpretable icons is highly challenging since individual interpretation can be hardly anticipated and formalized into rule sets [10]. In our study, 29.3\% of the test persons stated that symbols should be clearer and self-explanatory (unambiguous, colored, large symbols using pictograms), 15.5\% that symbols should be standardized, $15.5 \%$ that help should be offered in using the symbols (help button, detailed description in a separate help dialogue, tool-tips), and $10.3 \%$ that the number of tools and symbols should be reduced.

To eliminate the issue of ambiguity, additional assistance may be offered. One possibility would be to use a hint which appears when the user moves the mouse over the icon. In one study, it was found that the subjects expected such a tooltip [10]. The displayed information must be clear and concise. Furthermore, the window for the note should not be too big and not remain open for too long, otherwise another area of the application might be covered. Another important aspect in terms of assistance is the communication with the user: Practically speaking, the icons should change when the mouse is over them. For this purpose, the mouse could, for instance, take the form of a hand, cast a shadow, or the symbol could change its color.

Furthermore, users expect that the symbols are grouped in a clearly understandable way and not scattered over the entire screen. Through purposeful grouping, users will be supported in their operation. Another essential factor is the action that needs to take place immediately after the selection of a symbol. For example, after selecting a zoom-in button, users expect that the function is executed immediately without any further interaction. Generally speaking, it can be stated that current geo-portals are well-designed in this regard, as $76.0 \%$ of our study's test persons indicated that the order and the placement of the symbols support them in performing their tasks.

\subsubsection{Other}

Finally, other aspects that are essential to user experience in map-based geo-portals comprise a number of rather generic criteria.

First, long loading times are one of the main reasons for declining satisfaction in using geo-portals. ISO 9241 proposes in Part 11 [14] in accordance with the aspect of self-descriptiveness, that the system should point the user to longer loading times using interactive messages. This ensures that communication between the user and the system is intensified, thus making system processes more transparent to the user and consequently increasing satisfaction.

Another widely discussed aspect is scrolling, which users will only perform reluctantly in web-based interfaces. Accordingly, the use of scroll bars should be avoided [25]. All important information should be visible at a glance and not only be discovered by scrolling. This guarantees that users get a 
comprehensive overview of available information and datasets, and they are less likely to miss important information. Similarly, long legends should be avoided. This issue can be tackled by the use of folded groups displaying a generic term that represents a number of subsumed pieces of information. However, hierarchical structures like legends or layer trees should be limited to two levels to preserve comprehensibility.

A common shortcoming of many current geo-portals is that they open in a pop-up window-as a whole or for displaying the legend. Such windows are to be avoided regardless of the purpose they are used for. This is mainly due to the fact that most users have a strict aversion against pop-up windows as they are often used for advertising or gaming links. Most users indicate that they will close such windows immediately, in most cases before the content is fully loaded. Furthermore, many browsers block pop-up windows by default, so the user first needs to change the according settings manually. Furthermore, there are several additional issues that arise with the use of pop-up windows:

- The working environment is restricted.

- Additional windows have to be manually closed by the user.

- Returning to the original page is impeded.

- The current window is obscured.

- They can be used in exceptional cases (e.g., for displaying PDF documents).

In addition, geo-portal users feel massively disrupted by advertising elements. The use of interactive advertising is seen as particularly disturbing as it compromises the user's focus and concentration in performing their professional tasks. In addition, elements like animated graphics, inactive links, outdated information [26], and flashing and moving text should be avoided.

The above Sub-Sections 5.1 and 5.2 contain a basic description of the single parameters and their significance for the use case of map-based geo-portals. The illustration of our design considerations for map-based geo-portals is descriptive and has not been validated in a separate user study as the goal of this paper is the establishment of a core set of user experience design guidelines and not the evaluation of their implementation in the course of a particular application.

\section{Conclusion}

We have recently been witnessing the growing establishment of map-centered web-based geo-portals on national, regional and local levels for the professional use of geo-data. However, a particular issue with these geo-portals is that each instance has been implemented in different ways in terms of design, usability, functionality, interaction possibilities, map size and symbologies. On the one hand, this heterogeneity in geo-portal developments is rooted in geospatial companies' unique design; on the other hand, and more importantly, it is due to lacking consistent guidelines for implementing map-based geo-portals. This also results from the fact that the term "geo-portal" has not been sufficiently defined so far.

Furthermore, most current geo-portal developments focus on functionality and technology rather than on the users and their needs. Consequently, only few surveys have been carried out that focus on map-based web pages and their requirements [5], and this is particularly true for web-based geo-portals. As a result, experiences in assessing the specific requirements of map-based geo-portals 
are rare. Particularly, no overall study has been carried out considering all key aspects of map-based geo-portals (map, search, presentation of search results, symbologies, and other aspects). In fact, previous approaches lack the investigation of the whole breadth of the topic of map-based geo-portals. Consequently, no consistent and uniform guidelines have been developed so far.

In this paper, we try to tackle these shortcomings by analyzing and formalizing the requirements for professional map-based geo-portals. This is done by defining the term "geo-portal" (see Sub-Section 3.1, answering research Question 1), by assessing functional requirements of a geo-portal, by analyzing previous geo-portal developments and by conducting an empirical user study to assess practically-oriented user requirements (Section 4, answering research Question 2), and finally by establishing a set of user experience design guidelines for the creation of map-based geo-portals (Section 5, answering research Question 3).

We define the term of a map-based "geo-portal" as a web-based system that allows users to discover particular geo-datasets by looking into the associated metadata, to portray the data on a map, and to retrieve the data in adequate formats to further process them in a professional workflow.

Our user study (see Section 4) involving 105 professional geo-portal users showed that the participants have clearly qualifiable requirements that need to be thoroughly addressed. These requirements concern (1) user experience related parameters; and (2) design-related parameters.

As a result from the user study, we defined the user experience relevant parameters (see Sub-Section 5.1): a typical geo-portal user in terms of different user experience relevant parameters including the users themselves (pre-knowledge, experience, usage context), the goals a user needs to achieve (tasks to perform), the context (physical context, the technical context, and the social context), learnability (how easy it is for users to perform the defined tasks the first time), efficiency and effectiveness (how quickly and how effectively users can perform a certain task), memorability (how easy it is for users to re-establish their proficiency when they re-use an application), errors (how many errors users make and how these errors affect usage of an application), and satisfaction (how subjectively pleasant it is for users to use the application).

Furthermore, we derived a set of key considerations for user experience design in geo-portals (see Sub-Section 5.2). These design guidelines have been extracted for each of the main components including a geo-portal's map, search dialogues, presentation of search results, symbologies, and other aspects such as performance, the use of pop-up windows or scrolling. These guidelines shall constitute the basis for future geo-portal developments to achieve standardization in the user-experience design of map-based geo-portals.

\section{Acknowledgments}

The authors would like to thank all involved research groups at the Harvard Center for Geographical Analysis, the MIT SENSEable City Lab and at University of Heidelberg for offering their valuable thoughts. Furthermore, a special thanks goes to the test persons who helped in the evaluation and improvement of our research. This research has been supported by the Klaus Tschira Stiftung gGmbH. Finally, the authors would like to thank Jens Schaefermeyer from WhereGroup GmbH for his support in conceiving the research questions and requirements analysis. 


\section{Conflicts of Interest}

The authors declare no conflict of interest.

\section{References}

1. European Parliament and the Council. Directive 2007/2/EC of the European Parliament and of the Council of 14 March 2007 Establishing an Infrastructure for Spatial Information in the European Community (INSPIRE). Available online: http://inspire.jrc.ec.europa.eu (accessed on 15 October 2012).

2. Tait, M.G. Implementing geoportals: Applications of distributed GIS. Comput. Environ. Urban Syst. 2005, 29, 33-47.

3. Aditya, T.; Kraak, M.J. Reengineering the Geoportal: Applying HCI and Geovisualization Disciplines. In Proceedings of the 11th EC-GI \& GIS Workshop, Alghero, Italy, 29 June-1 July 2005.

4. Watanabe, M.; Yonemura, S.; Asano, Y. Investigation of Web Usability Based on the Dialogue Principles. In Human Centered Design; Kurosu, M., Ed.; Springer Berlin-Heidelberg: Berlin, Germany, 2009; pp. 825-832.

5. Nivala, A.M.; Brewster, S.; Sarjakoski, L.T. Usability evaluation of web mapping sites. Cartogr. J. 2008, 45, 129-138.

6. Skarlatidou, A.; Haklay, M. Public Web Mapping: Preliminary Usability Evaluation. In Proceedings of GIS Research UK Conference, Nottingham, UK, 5-7 April 2006.

7. Hornbaek, K.; Bederson, B.B.; Plaisant, C. Navigation patterns and usability of zoomable user interfaces with and without an overview. ACM Trans. Comput. Hum. Interact. 2002, 9, 362-389.

8. Harrower, M.; Sheesley, B. Designing better map interfaces: A framework for panning and zooming. Trans. GIS 2005, 9, 77-89.

9. You, M.; Chen, C.W.; Liu, H.; Lin, H. A usability evaluation of web map zoom and pan functions. Int. J. Des. 2007, 1, 15-25.

10. Kramers, R.E. Interaction with maps on the internet-A user centered design approach for the atlas of Canada. Cartogr. J. 2008, 45, 98-107.

11. He, X.; Persson, H.; Östman, A. Geoportal usability evaluation. Int. J. Spat. Data Infrastruct. Res. 2012, 7, 88-106.

12. Danko, D.M. Geospatial Metadata. In Springer Handbook of Geographic Information; Kresse, W., Danko, D.M., Eds.; Springer Berlin-Heidelberg: Berlin, Germany, 2012; pp. 359-392.

13. Nielsen, J. Usability Engineering; Academic Press: Boston MA, USA, 1993.

14. Part 11 Guidance on Usability. In ISO 9241 Ergonomics of Human-System Interaction; International Organization for Standardization: Geneva, Switzerland, 2006.

15. Adikari, S.; McDonald, C.; Campbell, J. A Design Science Framework for Designing and Assessing User Experience. In Human-Computer Interaction; Jacko, J.A., Ed.; Springer Berlin-Heidelberg: Berlin, Germany, 2011; pp. 25-34.

16. Part 210 Human-centered Design for Interactive Systems. In ISO 9241 Ergonomics of Human-System Interaction; International Organization for Standardization: Geneva, Switzerland, 2010. 
17. Geis, T. Usability und User Experience Unterscheiden. 2012. Available online: http://blog.procontext.com/2010/03/usability-und-user-experienceunterscheiden.html (accessed on 19 July 2013).

18. Resch, B.; Zipf, A.; Breuss-Schneeweis, P.; Beinat, E.; Boher, M. Towards the live city-Paving the way to real-time urbanism. Int. J. Adv. Int. Sys. 2012, 5, 470-482.

19. Resch, B.; Hillen, F.; Reimer, A.; Spitzer, W. Towards 4D cartography_Four-dimensional dynamic maps for understanding spatio-temporal correlations in lightning events. Cartogr. J. 2013, 3, 266-275.

20. Çöltekin, A.; Heil, B.; Garlandini, S.; Fabrikant, S.I. Evaluating the effectiveness of interactive map interface designs: A case study integrating usability metrics with eye-movement analysis. Cartogr. Geogr. Inf. Sci. 2009, 36, 5-17.

21. Haklay, M.; Tobon, C. Usability evaluation and PPGIS: Towards a usercentered design approach. Int. J. Geogr. Inf. Sci. 2003, 17, 577-592.

22. Haklay, M.; Zafiri, A. Usability engineering for GIS: Learning from a screenshot. Cartogr. $J$. 2008, 45, 87-97.

23. Sagl, G.; Resch, B.; Mittlboeck, M.; Hochwimmer, B.; Lippautz, M.; Roth, C. Standardised geo-sensor webs and web-based geo-processing for near real-time situational awareness in emergency management. Int. J. Bus. Cont. Risk Man. 2013, 4, 339-358.

24. Nielsen, J.; Loranger, H. Web Usability; Addison-Wesley: Munich, Germany, 2006.

25. Fernández-Wyttenbach, A.; Moya-Honduvilla, J.; Álvarez, M.; Bernabé-Poveda, M. First Approaches to the Usability of Digital Map Libraries. In Proceedings of the 3rd International Workshop in Digital Approaches to Cartographic Heritage, Barcelona, Spain, 26-27 June 2008.

26. Resch, B.; Blaschke, T.; Mittlboeck, M. Live geography: Interoperable geo-sensor webs facilitating the vision of digital earth. Int. J. Adv. Net. Serv. 2010, 3, 323-332.

(C) 2013 by the authors; licensee MDPI, Basel, Switzerland. This article is an open access article distributed under the terms and conditions of the Creative Commons Attribution license (http://creativecommons.org/licenses/by/3.0/). 